\title{
Magnetic fields in O-type stars measured with FORS 1 at the VLT
}

\author{
Swetlana Hubrig ${ }^{1}$, M. Schöller ${ }^{2}$, R. S. Schnerr ${ }^{3}$, I. Ilyin ${ }^{4}$, \\ H. F. Henrichs ${ }^{5}$, R. Ignace ${ }^{6}$ and J. F. González ${ }^{7}$
}

${ }^{1}$ ESO, Santiago, Chile; email: shubrig@eso.org; ${ }^{2}$ ESO, Garching, Germany; ${ }^{3}$ Inst. for Solar Physics, Royal Swedish Academy of Sciences, Stockholm, Sweden: ${ }^{4}$ AIP, Potsdam, Germany;

${ }^{5}$ University of Amsterdam, The Netherlands ${ }^{6}$ East Tennessee State University, Johnson City, USA $;{ }^{7}$ Complejo Astronomico El Leoncito, San Juan, Argentina

\begin{abstract}
The presence of magnetic fields in O-type stars has been suspected for a long time. The discovery of such fields would explain a wide range of well documented enigmatic phenomena in massive stars, in particular cyclical wind variability, $\mathrm{H} \alpha$ emission variations, chemical peculiarity, narrow X-ray emission lines and non-thermal radio/X-ray emission. Here we present the results of our studies of magnetic fields in O-type stars, carried out over the last years.
\end{abstract}

Keywords. Stars: early-type - stars: magnetic fields - techniques: polarimetric - stars: HD 36879, HD 148937, HD 152408, HD 164794, HD 191612

\section{Introduction}

Direct measurements of the magnetic field strength in massive stars using spectropolarimetry to determine the Zeeman splitting of the spectral lines are difficult, since only a few spectral lines are available for these measurements, which are usually strongly broadened by rapid rotation. Before our study (Hubrig et al. 2008), a magnetic field had only been found in the three O-type stars, $\theta^{1}$ Ori C, HD 155806, and HD 191612 (Donati et al. 2002; Hubrig et al. 2007; Donati et al. 2006).

\section{Observations and analysis}

More than 50 spectropolarimetric observations of 15 O-type stars were obtained from 2005 to 2008 using the multi-mode instrument FORS 1 at the 8.2-m Kueyen telescope. GRISMs $600 \mathrm{~B}$ and $600 \mathrm{R}$ were used with the $0.4^{\prime \prime}$ slit to obtain $R \approx 2000$ and $R \approx 3000$, respectively. Longitudinal magnetic fields were measured in two ways: using only the absorption hydrogen Balmer lines or using the entire spectrum including all available absorption lines of hydrogen, He I, He II, C III, C IV, N II, N III, and O III. Most of the targets were observed on three or four different nights to take into account the strong dependence of the longitudinal magnetic field on rotational aspect. Interestingly, a large part of the observed stars exhibited a change of polarity over certain nights. Four stars of our sample, HD 36879, HD 148937, HD 152408, and HD 164794, showed evidence for the presence of a weak magnetic field in the measurements using all spectral absorption lines. The uncertainties in the mean longitudinal field determination are obtained from the formal uncertainty in the linear regression of $V / I$ versus the quantity $-\frac{g_{\text {eff }} e}{4 \pi m_{e} c^{2}} \lambda^{2} \frac{1}{I} \frac{\mathrm{d} I}{\mathrm{~d} \lambda}\left\langle B_{z}\right\rangle+V_{0} / I_{0}$. 


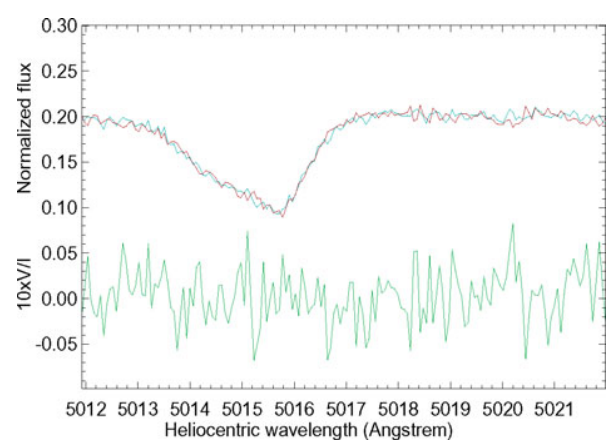

Figure 1. Low signal-to-noise Stokes $I$ and $V$ spectra of HD 191612 obtained with the echelle spectrograph SOFIN. Due to the rather fast rotation of the star the contribution of blends is not easily recognizable in the Stokes $I$ spectrum. On the other hand, the blends become detectable in the Stokes $V$ spectrum due to the Zeeman signatures produced in magnetically sensitive lines.

\section{Discussion}

This is the first time that magnetic field strengths were determined for such a large sample of stars, with an accuracy comparable to the errors obtained for the three previously known magnetic O-type stars, $\theta^{1}$ Ori C, HD 155806, and HD 191612. For the magnetic Of?p star HD 191612, Donati et al. (2006) measured a magnetic field of $\left\langle B_{\mathrm{z}}\right\rangle=$ $-220 \pm 38 \mathrm{G}$, by averaging a total of 52 exposures obtained over 4 different nights. This is similar to our typical errors of a few tens of $\mathrm{G}$. The new high-resolution observations $(R \approx 30000)$ of the $\mathrm{O} 7 \mathrm{~V}(\mathrm{n})$ star HD 36879 and the O8fpe star HD 191612 with the SOFIN echelle spectrograph mounted at the $2.56 \mathrm{~m}$ Nordic Optical Telescope indicate the presence of weak magnetic fields of positive polarity in both stars. In spite of a rather low signal-to-noise ratio achieved in these observations $(\mathrm{S} / \mathrm{N} \approx 200-270)$, caused by bad weather conditions, it was still possible to detect Zeeman features at the positions of He II, C IV, O II, and N III lines. An example of our observation of HD 191612 is presented in Fig. 1.

The four new magnetic O-type stars have different spectral types, luminosity classes, and behavior in various observational domains. The study of the evolutionary state of one of the Galactic Of?p stars, HD 191612, indicates that it is significantly evolved with an $\sim 08$ giant-like classification (Howarth et al. 2007). The youth of the most carefully studied magnetic O-type star $\theta^{1}$ Ori $\mathrm{C}$ and the older age of the Of?p star HD 191612 suggest that the presence of magnetic fields in O-type stars is unrelated to their evolutionary state. We note that it is unclear yet whether more complex, smaller scale fields play a role in the atmospheres of hot stars. In the case of a more complex magnetic field topology, the longitudinal magnetic field integrated over the visible stellar surface will be smaller (or will even cancel) and will not be easily detected with the low-resolution FORS 1 measurements. However, high resolution spectropolarimeters (like ESPaDOnS, Narval, or SOFIN) should be able to detect such complex fields.

\section{References}

Donati, J.-F., Babel, J., Harries, T. J., et al. 2002, MNRAS 333, 55

Donati, J.-F., Howarth, I. D., Bouret, J.-C., et al. 2006, MNRAS 365, L6

Howarth, I. D., Walborn, N. R., Lennon, D. J., et al. 2007, MNRAS 381, 433

Hubrig, S., Yudin, R. V., Pogodin, M., et al. 2007, AN 328, 1133

Hubrig, S., Schöller, M., Schnerr, R. S., et al. 2008, A\&SA 490, 793 